\title{
Prospero homeobox 1 mediates the progression of gastric cancer by inducing tumor cell proliferation and lymphangiogenesis
}

\author{
Kang-Jin Park ${ }^{1} \cdot$ Sung-Bum Cho ${ }^{1} \cdot$ Young-Lan Park $^{1} \cdot$ Nuri Kim $^{1} \cdot$ \\ Sun-Young Park ${ }^{1} \cdot$ Dae-Seong Myung ${ }^{1} \cdot$ Wan-Sik Lee $^{1} \cdot$ Sun-Seog Kweon $^{2}$. \\ Young-Eun Joo ${ }^{1}$
}

Received: 18 October 2015/Accepted: 21 December 2015/Published online: 12 January 2016

(c) The International Gastric Cancer Association and The Japanese Gastric Cancer Association 2016

\begin{abstract}
Background Prospero homeobox 1 (PROXI) functions as a tumor suppressor gene or an oncogene in various cancer types. However, the distinct function of PROXI in gastric cancer is unclear. We determined whether PROX1 affected the oncogenic behavior of gastric cancer cells and investigated its prognostic value in patients with gastric cancer. Methods A small interfering RNA against PROXI was used to silence PROXI expression in gastric cancer cell lines AGS and SNU638. Expression of PROX1 in gastric cancer tissues was investigated by performing immunohistochemistry. Apoptosis, proliferation, angiogenesis, and lymphangiogenesis were determined by performing the TUNEL assay and immunohistochemical staining for Ki67, CD34, and D2-40.

Results PROXI knockdown induced apoptosis by activating cleaved caspase-3, caspase-7, caspase-9, and poly(ADP-ribose) polymerase, and by decreasing the expression of anti-apoptotic proteins Bcl-2 and Bcl-xL. PROX1 knockdown also suppressed tumor cell proliferation. In addition, $P R O X 1$ knockdown decreased lymphatic
\end{abstract}

Kang-Jin Park and Sung-Bum Cho have contributed equally to this work.

Electronic supplementary material The online version of this article (doi:10.1007/s10120-015-0592-y) contains supplementary material, which is available to authorized users.

Young-Eun Joo

yejoo@chonnam.ac.kr

1 Department of Internal Medicine, Chonnam National University Medical School, 8 Hak-Dong, Dong-ku, Gwangju 501-757, Republic of Korea

2 Department of Preventive Medicine, Chonnam National University Medical School, Gwangju, Republic of Korea endothelial cell invasion and tube formation and the expression of vascular endothelial growth factor (VEGF)-C and -D and cyclooxygenase (COX)-2. However, PROXI knockdown only decreased umbilical vein endothelial cell invasion, not tube formation. The mean Ki-67 labeling index and lymphatic vessel density value of PROX1-positive tumors were significantly higher than those of PROX1-negative tumors. However, no significant difference was observed between PROX1 expression and apoptotic index or microvessel density. PROX1 expression was significantly associated with age, cell differentiation, lymph node metastasis, cancer stage, and poor survival.

Conclusions These results indicate that PROX1 mediates the progression of gastric cancer by inducing tumor cell proliferation and lymphangiogenesis.

Keywords Prospero homeobox 1 - Proliferation · Lymphangiogenesis · Prognosis - Stomach neoplasm

\section{Introduction}

Gastric cancer is one of the major causes of cancer-related morbidity and mortality worldwide. Despite much progress in therapeutic techniques for treating gastric cancer, clinical outcomes in patients with advanced gastric cancer are poor, with cancer metastasis being the major cause of cancer-related morbidity and mortality [1, 2]. Studies investigating molecular and biochemical changes in gastric cancer indicate that metastasis of gastric cancer is a complex process caused by mutations that alter the functions of proto-oncogenes and tumor suppressor genes, resulting in deregulated proliferation and differentiation of cells, inhibition of apoptosis, increased invasiveness, 
perturbation of cell adhesion, promotion of angiogenesis, and inhibition of immune surveillance [3-6].

Homeobox genes encode transcription factors that control cell proliferation, differentiation, and morphogenesis during embryonic development [7]. Prospero homeobox 1 (PROX1) is a homeobox gene that is related to the Drosophila prospero gene and plays a critical role in determining cell fate during the development of various organs, including neuronal differentiation in the central nervous system, elongation of lens fibers, migration of hepatocytes, differentiation of retinal cells, stabilization of myocardial actin, pancreatic growth, and induction of lymphatic endothelial cells [8].

PROX1 is aberrantly expressed in various human cancers, suggesting its role in carcinogenesis [8]. PROXI plays multifunctional roles in human cancers and acts as a tumor suppressor gene or an oncogene based on the cancer type, thus exerting variable effects on cell proliferation and differentiation [8]. Decreased expression of PROX1 is observed in pancreatic cancers, hepatocellular carcinomas, sporadic breast cancers, and biliary carcinomas, indicating that $P R O X I$ plays a tumor-suppressive role in these cancers [9-12]. In contrast, overexpression of PROXI is observed in colorectal cancers, endothelial tumors, and gliomas, indicating that $P R O X 1$ promotes the progression of these cancers [13-16]. These data suggest that PROXI could be a potential molecular target for treating human cancers. However, it is unclear whether PROXI exerts tumor-suppressive or oncogenic effects in gastric cancer.

The present study investigated the possible role of PROX1 in gastric carcinogenesis and examined its correlation with the clinicopathological features of gastric cancer, including prognosis.

\section{Materials and methods}

\section{Cell lines, culture, and siRNA transfection}

Human gastric cancer cell lines AGS and SNU638 (ATCC, Manassas, VA, USA) were grown in RPMI-1640 (HyClone, Loan, UT, USA) supplemented with $10 \%$ fetal bovine serum and antibiotics. Transfection of cells with PROXI siRNA (BIONEER, Daejeon, Korea) and scrambled siRNA (Qiagen, Germantown, MD, USA) was performed using Lipofectamine ${ }^{\mathrm{TM}}$ RNAiMAX (Invitrogen, Carlsbad, CA, USA), according to the manufacturer's recommendations. Human umbilical vein endothelial cells (HUVECs; Lonza, Walkersville, MD, USA) and human lymphatic endothelial cells (HLECs; Sciencell, San Diego, CA, USA) were cultured in $\mathrm{EBM}^{\mathrm{TM}}-2$ supplemented with EGM $^{\mathrm{TM}}-2$ SingleQuot ${ }^{\mathrm{TM}}$ Kit (Lonza).

\section{Western blotting}

Cell lysates were prepared by sonicating cells in RIPA ${ }^{\circledR}$ reagent (Thermo, Rockford, IL, USA) for $15 \mathrm{~s}$. Proteins were separated by performing sodium dodecyl sulfatepolyacrylamide gel electrophoresis and were electrotransferred onto a PVDF membrane (Millipore, Billerica, MA, USA). Specific proteins were detected using an enhanced chemiluminescence western blotting kit (Millipore) and a luminescent image analyzer LAS-4000 (Fujifilm, Tokyo, Japan). Antibodies against the following proteins were used in this study: human PROX1 (OriGene, Rockville, MD, USA); cleaved caspases (caspase-3, caspase-7, and caspase-9) and poly(ADP-ribose) polymerase (PARP), Bcl2, Bcl-xL, Bax, Bid, hypoxia-inducible factor- $1 \alpha(\mathrm{HIF}-1 \alpha)$, E-cadherin, zonula occludens-1 (ZO-1), snail, vimentin, extracellular signal-regulated kinase (ERK), phosphorylated ERK, p38, phosphorylated p38, c-Jun NH2-terminal kinase (JNK), phosphorylated JNK, phosphorylated ERK1/ 2 , phosphorylated $\beta$-catenin, and $\beta$-catenin (Cell Signaling, Danvers, MA, USA); vascular endothelial growth factor-A (VEGF-A), VEGF-C, VEGF-D, cyclooxygenase-2 (COX2 ), and $\beta$-tubulin (Santa Cruz Biotechnology, CA, USA); and endostatin, angiostatin, matrix metalloproteinase-2 (MMP-2), and MMP-9 (Abcam, Cambridge, UK).

\section{Cell proliferation assay}

Proliferation of transfected cells was assessed using watersoluble tetrazolium salt (WST-1; Daeil Lab Inc., Seoul, Korea). Transfected cells were seeded at a density of $1 \times 10^{4}$ cells/well in 96-well plates, incubated overnight, and treated with WST- 1 at $37^{\circ} \mathrm{C}$. Cell proliferation was assessed by measuring the absorbance at $450 \mathrm{~nm}$ using a microplate reader.

\section{Flow cytometric analysis}

Transfected cells were detached by trypsinization, washed with PBS, and resuspended in binding buffer (BD Biosciences, San Diego, CA, USA). Cell suspensions were stained with APC Annexin V and 7-amino-actinomycin D (BD Biosciences) for $20 \mathrm{~min}$ at RT. Apoptotic cells were analyzed using BD Cell Quest ${ }^{\circledR}$ version 3.3 (Becton, Dickinson and Company, San José, CA, USA) and WinMDI version 2.9 (The Scripps Research Institute, San Diego, CA, USA).

\section{Matrigel invasion assay}

For the Matrigel invasion assay, HUVECs and HLECs were inoculated in the upper chamber of a $1 \mathrm{mg} / \mathrm{ml}$ Matrigel-coated Transwell chamber $(8-\mu \mathrm{m}$ pore; Corning 
Costar, Corning, NY, USA). The lower chamber was filled with cell-free conditioned media $(\mathrm{CM})$ from transfected AGS and SNU638 cells. After incubating for $16 \mathrm{~h}$, cells invading the lower surface of the Matrigel-coated Transwell membrane were stained with Diff-Quik (Sysmex, Kobe, Japan) and counted.

\section{In vitro endothelial tube formation assay}

HUVECs and HLECs were trypsinized, seeded in Matrigel (BD Bioscience)-coated 96-well plates, and incubated with cell-free conditioned media (CM) from transfected AGS and SNU638 cells for $16 \mathrm{~h}$. Degree of tube formation was photographed using an inverted microscope and calculated using the WIMTube image analysis platform (WIMASIS GmbH, Munich, Germany).

\section{Cell adhesion assay}

Cell adhesion assay was conducted by coating a 96-well plate with fibronectin, collagen I, and collagen IV. Nonspecific binding sites in the plate were blocked using $0.2 \%$ bovine serum albumin (BSA) in PBS. SiRNA-transfected AGS and SNU638 cells cultured in serum-free media were plated in the coated 96-well plate and incubated for $15 \mathrm{~min}$. Nonadherent cells were removed by gentle agitation, and adherent cells were assessed by performing the WST-1 assay.

\section{Transwell migration assay}

The in vitro migratory potential of the gastric cancer cells was examined using 24-well Transwell plates containing polycarbonate filter inserts with a pore size of $8 \mu \mathrm{m}$ (Corning Costar). SiRNA-transfected AGS and SNU638 cells cultured in $0.2 \%$ BSA were plated in the upper chamber of non-coated Transwell plates, and the lower chamber was filled with $10 \%$ BSA. After incubation for $16 \mathrm{~h}$, cells that had migrated to the lower membrane were stained with Diff-Quik and counted.

\section{Immunohistochemical analysis}

For immunostaining, paraffinized sections of tumor tissues from patients were dewaxed and were gradually rehydrated. Endogenous peroxidase activity was blocked using a peroxidase-blocking solution, and the sections were incubated with polyclonal rabbit anti-human PROX1 (Santa Cruz Biotechnology), anti-Ki-67 (Dakopatts, Glostrup, Denmark), anti-CD34 (Abcam), and anti-D2-40 (Dakopatts) antibodies. The sections were then stained using a Dako Real ${ }^{\mathrm{TM}}$ Envision HRP/DAB detection system
(Dako Cytomation, Glostrup, Denmark) and were counterstained using Mayer's hematoxylin (Sigma-Aldrich, St. Louis, MO, USA).

\section{Patients and tumor specimens}

Paraffin-embedded tissue samples used in this study were obtained from 327 consecutive patients with gastric cancer who underwent surgery between January 1999 and December 2000 at the Chonnam National University Hwasun Hospital (Jeonnam, Korea). None of the patients received preoperative chemotherapy or radiotherapy. Clinicopathological parameters at the time of the surgery were retrieved from medical records. Tumor staging was performed in accordance with the American Joint Committee on Cancer staging system [17]. Survival was measured from the time of surgery until follow-up on December 31, 2013. This study was approved by the Institutional Review Board of the Chonnam National University Hwasun Hospital. Also, ethical approval was obtained from the Institutional Review Board of the Chonnam National University Hwasun Hospital.

\section{Evaluation of PROX1 expression}

Immunostaining score of PROX1 was evaluated by 2 observers who were blinded to the clinicopathological data, and the score was determined by multiplying the stained area with the intensity. Stained area was graded on a scale of $0-3$ : 0 , none; $1,<10 \% ; 2,10-50 \%$; and 3 , $>50 \%$. Staining intensity was also graded on a scale of $0-3$ : 0 , no staining of cancer cells; 1 , weak staining of cancer cells; 2, moderate staining of cancer cells; and 3, strong staining of cancer cells. Samples with a total score of $\geq 6$ were designated PROX1-positive samples, while those with a total score of $<6$ were designated PROX1negative samples.

\section{Determination of tumor cell proliferation and apoptosis}

Proliferation of tumor cells was determined by immunostaining with anti-Ki-67 antibody. Ki-67 labeling index (KI) was defined as the number of $\mathrm{Ki}$-67-positive nuclei per 1000 tumor cell nuclei. Apoptotic cells were detected using the DeadEnd ${ }^{\mathrm{TM}}$ colorimetric terminal deoxynucleotidyl transferase dUTP nick-end labeling (TUNEL) system (Promega, Madison, WA, USA), according to the manufacturer's instructions. Apoptotic index (AI) was expressed as the number of TUNEL-positive nuclei containing an apoptotic body per 1000 tumor cell nuclei. 
A
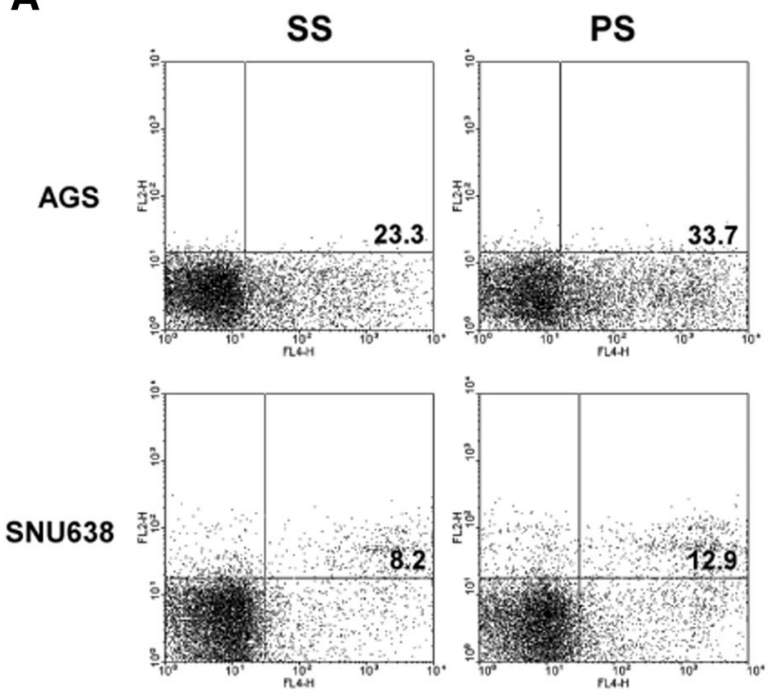

C

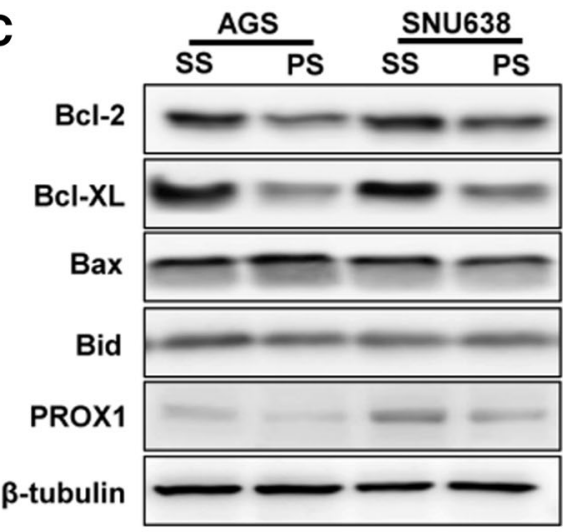

D

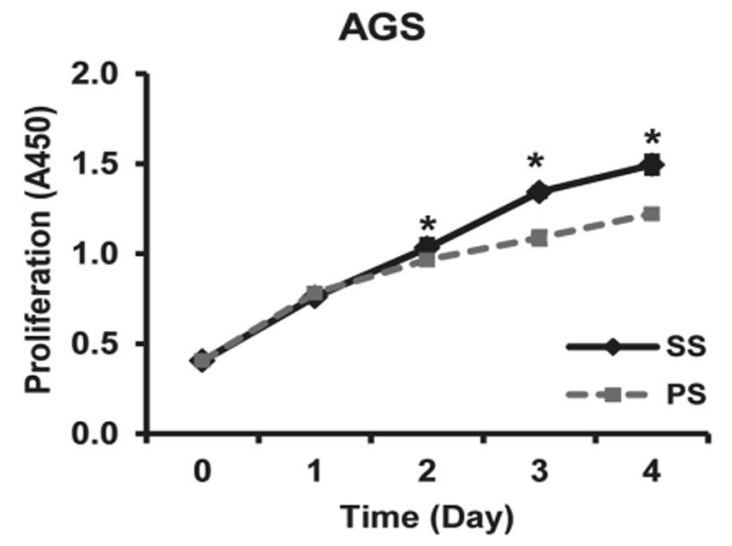

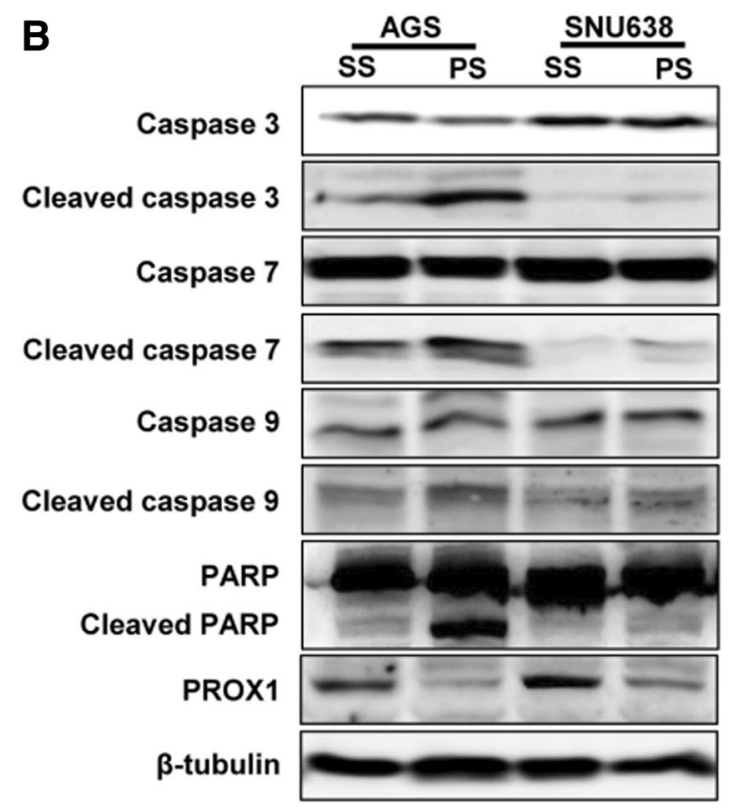

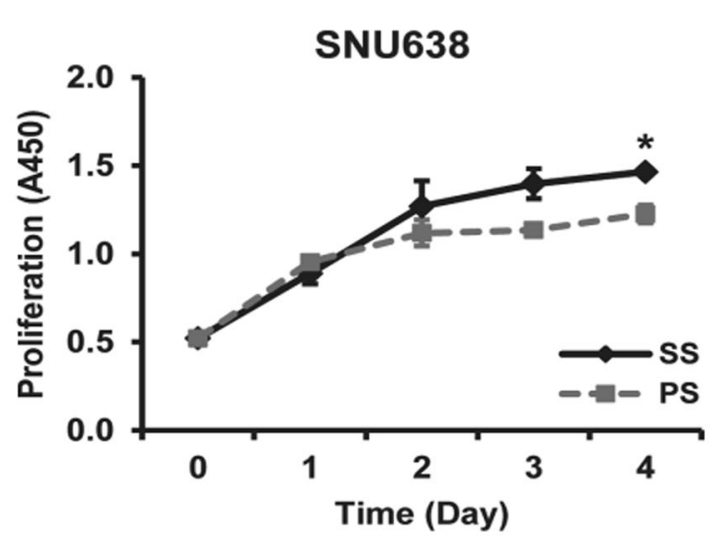


4Fig. $1 P R O X 1$ knockdown induces the apoptosis and inhibits the proliferation of human gastric cancer cells. a The proportion of early apoptotic cells was higher among PS-transfected AGS and SNU638 cells than among SS-transfected AGS and SNU638 cells (33.7 vs. $23.3 \%$ and 12.9 vs. $8.2 \%$, respectively). b Expression of total and cleaved caspase- 3 , caspase-7, caspase-9, and PARP. PROX1 knockdown increased the expression of cleaved caspase-3, caspase-7, caspase-9, and PARP. c Expression of apoptosis regulatory proteins. PROX1 knockdown decreased the expression of anti-apoptotic proteins Bcl-2 and Bcl-xL but did not alter the expression of proapoptotic proteins Bax and Bid. Results are representative of 3 independent experiments. d PROX1 knockdown inhibits the proliferation of human gastric cancer cells. The proportion of proliferating cells, as determined by measuring absorbance, was significantly lower among PS-transfected AGS cells than among SS-transfected AGS cells on days 2,3 , and $4(P=0.047, P=0.001$, and $P=0.001$, respectively), and was significantly lower among PS-transfected SNU638 cells than among SS-transfected SNU638 cells on day 4 $(P=0.013)$. SS scrambled siRNA; PS PROX1 siRNA; PARP poly(ADP-ribose) polymerase

\section{Determination of microvessel and lymphatic vessel densities}

Microvessel density (MVD) and lymphatic vessel density (LVD) were measured using samples that yielded positive results for anti-CD34 and anti-D2-40 antibodies, respectively. To assess MVD and LVD, immunostained vessels were scanned under low magnification $(40 \times)$, and areas showing the highest concentrations of vessels (hot spots) in the tumor and peritumoral parenchyma were selected. Hot spots were chosen for each sample, and 5 hot spots were examined under high magnification $(200 \times)$. MVD and LVD are expressed as the mean of vessels from 3 hot spots.

\section{Statistical analysis}

The relationship between PROX1 expression and various clinicopathological parameters was assessed using the chi square $\left(\chi^{2}\right)$ test and Fisher's exact test. Differences in patient survival were estimated using the Kaplan-Meier method and were analyzed using the log-rank test. Survival data were evaluated using multivariate Cox regression analysis. The relationship between PROX1 expression and KI, AI, MVD, and LVD was evaluated using Student's $t$-test. All statistical analyses was conducted using the Statistical Package for the Social Sciences (SPSS/PC + version 15.0; Chicago, IL, USA), and $P \leq 0.05$ was considered statistically significant.

\section{Results}

\section{PROX1 knockdown induces the apoptosis of human gastric cancer cells}

We used PROX1 siRNA-transfected cells to evaluate the impact of PROX1 knockdown on the oncogenic behavior of human gastric cancer cells. Transfection of cells with PROXI siRNA decreased both mRNA and protein expression of PROX1 (data not shown). We performed flow cytometric analyses to evaluate the impact of PROXI knockdown on apoptosis. Transfection of AGS and SNU638 cells with $P R O X 1$ siRNA increased the proportion of early apoptotic cells compared with the transfection of these cells with scrambled siRNA (33.7 vs. $23.3 \%$ and 12.9 vs. $8.2 \%$, respectively; Fig. 1a). We investigated caspase activities to determine the activation of caspases, the key enzymes of apoptosis, after PROX1 knockdown. Expression of cleaved caspase-3, caspase-7, caspase-9, and PARP was upregulated in AGS and SNU638 cells after PROX1 knockdown (Fig. 1b). We further examined whether PROX1 knockdown-induced apoptosis was associated with the modulation of apoptosis-regulatory proteins. We observed that PROX1 knockdown decreased the expression of anti-apoptotic proteins Bcl-2 and Bcl-xL but did not alter the expression of pro-apoptotic proteins Bax and Bid (Fig. 1c).

\section{PROX1 knockdown inhibits the proliferation of human gastric cancer cells}

To determine the effects of PROX1 on cell proliferation, we performed a cell proliferation assay on days $1,2,3$, and 4 after the transfection of cells with PROX1 siRNA. The proportion of proliferating cells, as determined by measuring absorbance, was significantly lower among PROX1 siRNA-transfected AGS cells than among scrambled siRNA-transfected AGS cells on days 2, 3, and 4 ( $P=0.047, P=0.001$, and $P=0.001$, respectively $)$, and was significantly lower among $P R O X 1$ siRNA-transfected SNU638 cells than among scrambled siRNA-transfected SNU638 cells on day 4 ( $P=0.013$; Fig. 1d).

\section{Impact of $P R O X 1$ knockdown on the angiogenesis of human gastric cancer cells}

To evaluate the effect of PROX1 on the angiogenesis of HUVECs, we performed Matrigel invasion and tube formation assays by using conditioned media (CM) from PROX1 and scrambled siRNA-transfected AGS and SNU638 cells. Invasion of HUVECs cultured in CM from PROX1 siRNA-transfected AGS and SNU638 cells was significantly decreased compared with that of HUVECs cultured in CM from scrambled siRNA-transfected AGS and SNU638 cells $(P=0.017$ and $P=0.010$, respectively; see 1a in the Electronic supplementary material, ESM). However, CM from PROX1 siRNA-transfected AGS and SNU638 cells did not inhibit the formation of endothelial tubes compared with CM from scrambled siRNA-transfected AGS and SNU638 cells $(P=0.098$ and 
$P=0.255$, respectively; ESM 1b). PROX1 knockdown decreased the expression of the angiogenic inducers HIF$1 \alpha$ (in all the tested cells) and VEGF-A (in AGS cells), but did not alter the expression of the angiogenic inhibiters endostatin and angiostatin (in all the tested cells; ESM 1c).

\section{Impact of PROX1 knockdown on the lymphangiogenesis of human gastric cancer cells}

To evaluate the effects of PROX1 on the lymphangiogenesis of HLECs, we performed Matrigel invasion and tube formation assays using CM from PROXI and scrambled siRNA-transfected AGS and SNU638 cells. Invasion and tube formation of HLECs cultured in CM from PROX1 siRNA-transfected AGS $(P=0.034$ and $P=0.022$, respectively) and SNU638 cells $(P=0.020$ and $P=0.037$ respectively) were significantly decreased compared with those of HLECs cultured in CM from scrambled siRNAtransfected cells (Fig. 2a, b). PROXI knockdown decreased the levels of the lymphangiogenic inducers VEGF-C, VEGF-D, and COX-2 in all of the tested cells (Fig. 2c).

\section{Impact of PROX1 knockdown on epithelial to mesenchymal transition of human gastric cancer cells}

We performed cell adhesion and migration assays to investigate the relationship between PROX1 and epithelial to mesenchymal transition (EMT) in human gastric cancer cells. The cell adhesion ability of siRNA-transfected cells was measured using 3 cell adhesion substrates: fibronectin, collagen I, and collagen IV. PROXI siRNA-transfected AGS cells showed higher adhesion to collagen I and collagen IV than scrambled siRNA-transfected AGS cells ( $P=0.012$ and $P=0.001$, respectively), and PROXI siRNA-transfected SNU638 cells showed higher adhesion to fibronectin, collagen I, and collagen IV ( $P=0.013$, $P=0.018$, and $P=0.019$, respectively; Fig. 3a) than scrambled siRNA-transfected SNU638 cells. The number of migratory cells was significantly lower among the PROXI siRNA-transfected AGS and SNU638 cells than among scrambled siRNA-transfected cells $(P=0.037$ and $P=0.048$, respectively; Fig. 3b). The cell morphologies of PROXI siRNA- and scrambled siRNA-transfected cells were observed via phase-contrast microscopy. PROX1 siRNA-transfected AGS and SNU638 cells displayed cobblestone-like morphologic features and growth in clusters, unlike scrambled siRNA-transfected AGS and SNU638 cells (Fig. 3c). To investigate the phenotypic changes induced by EMT, expression of the EMT-associated proteins MMP-2, MMP-9, ZO-1, snail, E-cadherin, and vimentin was assessed. We observed that the expression of MMP-2, MMP-9, ZO-1, and vimentin was lower in PROX1 siRNA-transfected AGS and SNU638 cells than in scrambled siRNA-transfected cells. However, the expression of E-cadherin and snail was not altered in PROXI siRNA-transfected cells (Fig. 3d).

\section{Impact of PROX1 knockdown on oncogenic signaling pathways in human gastric cancer cells}

To examine whether PROX1 activated oncogenic signaling pathways in human gastric cancer cells, we examined the phosphorylation of $\beta$-catenin and MAPK signaling proteins by performing western blotting. PROXI knockdown increased the phosphorylation of $\beta$-catenin and decreased the phosphorylation of ERK1/2, p38, and JNK in AGS and SNU638 cells (see ESM 2).

\section{Associations of PROX1 with the clinicopathological variables of human gastric cancer}

To study the prognostic role of PROX1 in the progression of human gastric cancer, we investigated PROX1 expression immunohistochemically in formalin-fixed, paraffin-embedded tissue sections obtained from 327 patients with gastric cancer, and correlated our results with the clinicopathological data for these patients. PROX1 expression was increased in gastric cancer and metastatic lymph node tissues compared with that in normal gastric mucosa and non-metastatic lymph node tissues (see ESM 3). Immunostaining of PROX1 protein was predominantly identified in the cytoplasm of cancer cells and was not detectable in the tumor stroma (ESM 3). We analyzed the survival rates of patients with gastric cancer and the association between PROX1 expression and clinicopathological parameters of these patients. We observed that PROX1 expression was significantly associated with age, cell differentiation, cancer stage, and lymph node metastasis $(P<0.001, \quad P=0.001$, $P=0.012$, and $P=0.002$, respectively; Table 1). Moreover, the overall survival of patients with PROX1positive tumors was significantly lower than that of patients with PROX1-negative tumors $(P<0.001$; Fig. 4). The associations between the clinicopathological parameters of patients with gastric cancer and prognosis of gastric cancer are shown in Table 2. Patients with PROX1-positive tumors showed an elevated risk of death after adjustment for age, sex, and tumor size, with a hazard ratio $(95 \% \mathrm{CI})$ of $1.662(1.180-2.339)$. 
A
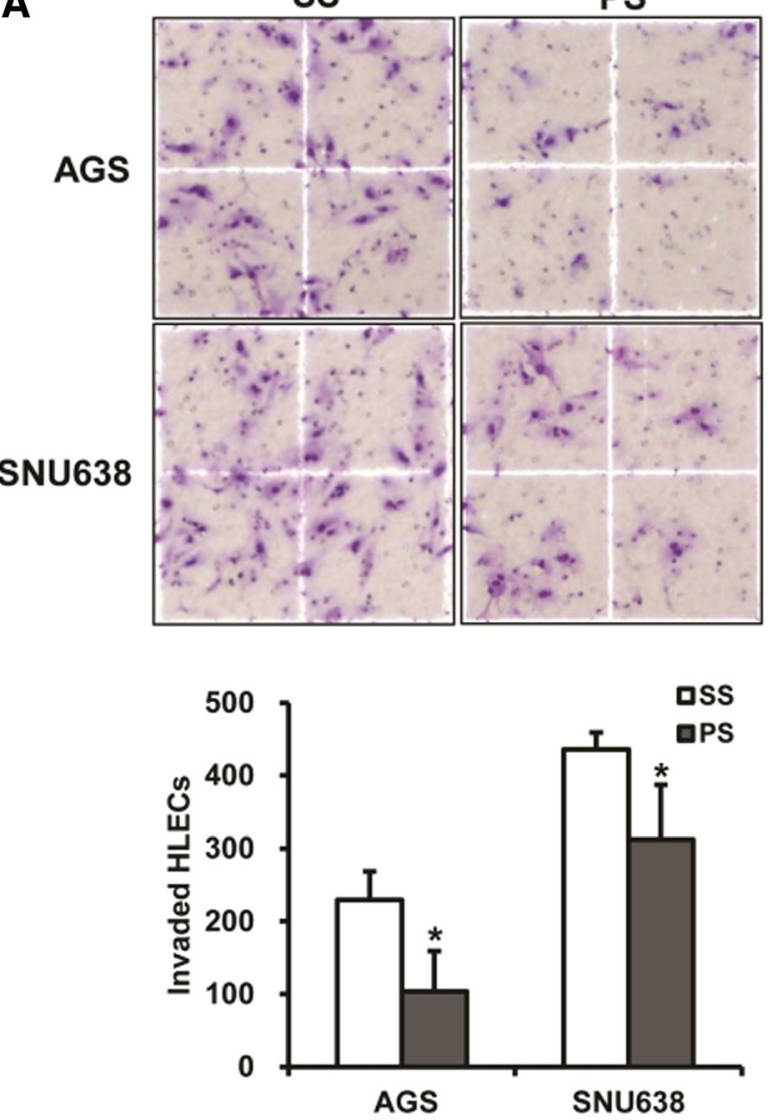

B
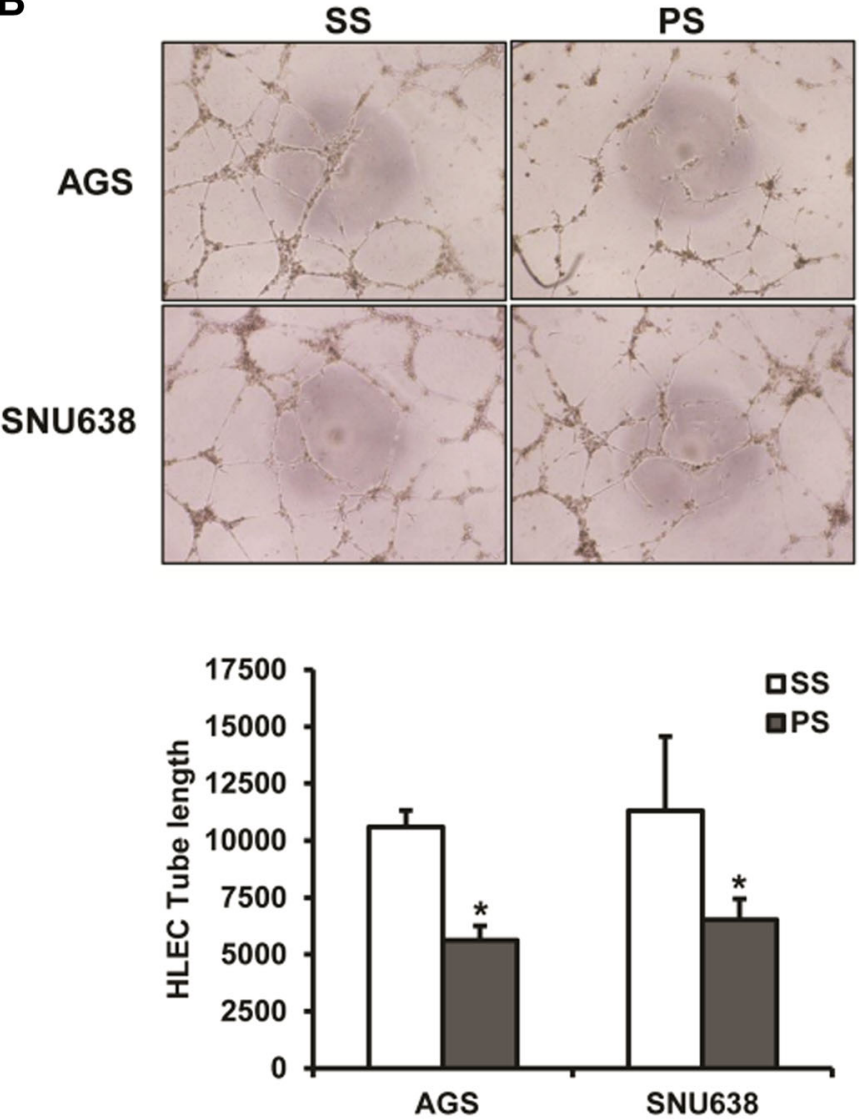

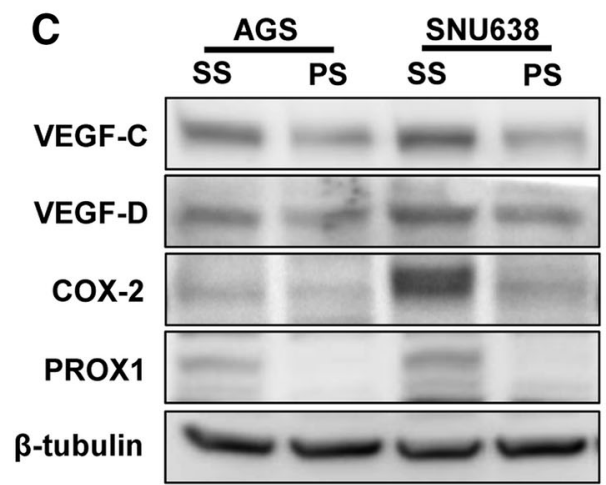

Fig. 2 Impact of PROXI knockdown on the lymphangiogenesis of human gastric cancer cells. a Invasion of HLECs cultured in CM from PS-transfected AGS and SNU638 cells was significantly decreased compared with that of HLECs cultured in CM from SS-transfected cells $(P=0.034$ and $P=0.020$, respectively). b CM from PStransfected AGS and SNU638 cells inhibited the formation of endothelial tubes compared with CM from SS-transfected AGS and

\section{Associations of PROX1 expression with apoptosis, proliferation, angiogenesis, and lymphangiogenesis of human gastric cancer cells}

All tumor samples were assessed by performing the TUNEL assay and via $\mathrm{Ki}-67, \mathrm{CD} 34$, and D2-40 immunostaining to determine the apoptosis, proliferation, angiogenesis, and
SNU638 cells $(P=0.022$ and $P=0.037$, respectively). c $P R O X 1$ knockdown decreased the expression of the lymphangiogenic inducers VEGF-C, VEGF-D, and COX-2 in all the tested cells. SS scrambled siRNA; PS PROXI siRNA; HLECs human lymphatic endothelial cells; $C M$ conditioned medium; $V E G F$ vascular endothelial growth factor; $C O X-2$ cyclooxygenase-2. ${ }^{*} P<0.05$ versus control

lymphangiogenesis of tumor cells (ESM 4). The AI of the 327 tumor samples ranged from 0.0 to 5.1, with a mean AI of $1.8 \pm 1.4$. No significant difference was observed between PROX1 expression and AI $(P=0.381)$. The KI of the 327 tumor samples ranged from 14.8 to 86.9 , with a mean KI of $49.8 \pm 17.8$. The mean KI value of PROX1-positive tumors was $60.0 \pm 15.9$, which was significantly higher than that of 


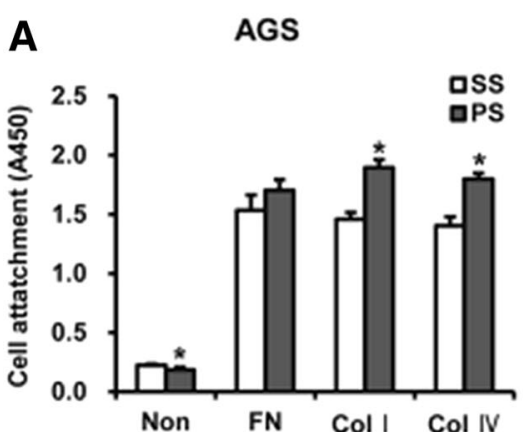

C

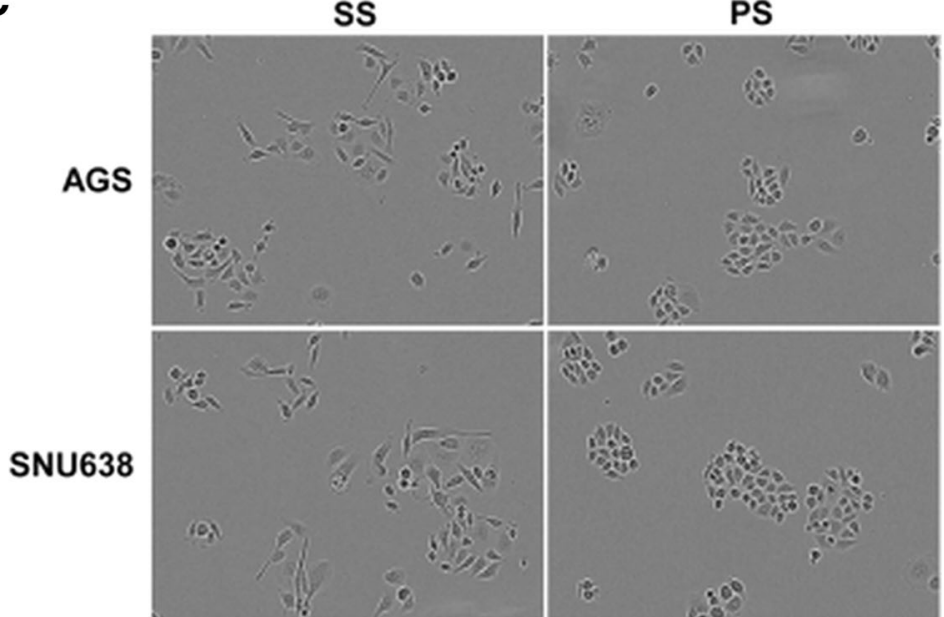

SNU638

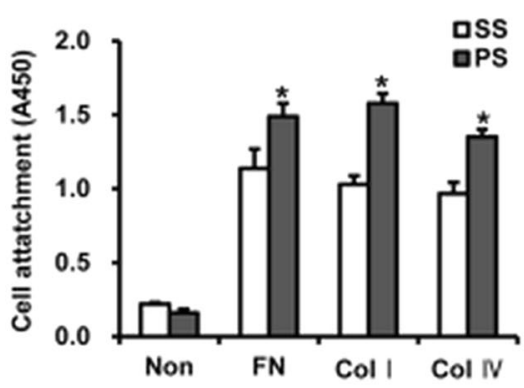

ass

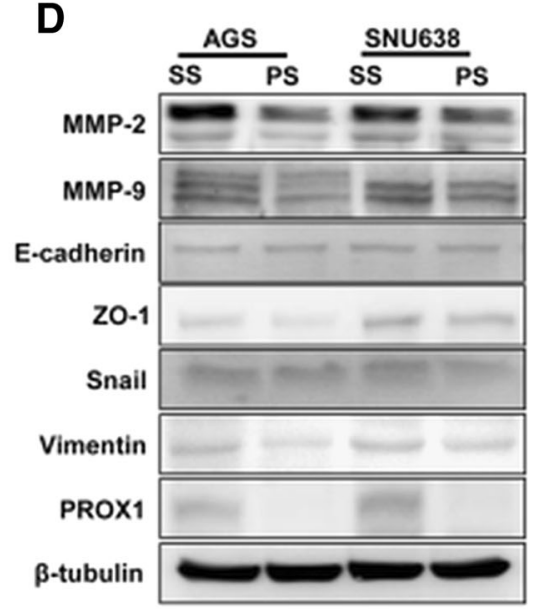

\section{B}

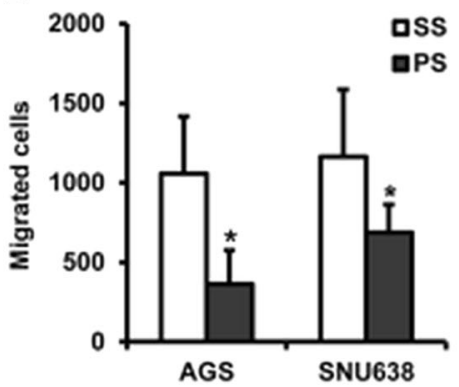

Fig. 3 Impact of PROX1 knockdown on the EMT of human gastric cancer cells. a The cell adhesion ability of siRNA-transfected gastric cancer cells was measured using 3 cell adhesion substrates: fibronectin, collagen I, and collagen IV. Adherent cells were performed using WST-1 assay, and quantified by measuring the absorbance at $450 \mathrm{~nm}$ using a plate reader. PS-transfected AGS cells showed higher adhesion to collagen I and collagen IV than SStransfected AGS cells $(P=0.012$ and $P=0.001$, respectively), and PS-transfected SNU638 cells showed higher adhesion to fibronectin, collagen I, and collagen IV than SS-transfected SNU638 cells ( $P=0.013, P=0.018$, and $P=0.019$, respectively). b The number of migratory cells was significantly lower among PS-transfected AGS

PROX1-negative tumors $(P=0.002)$. The MVD of the 327 tumor samples ranged from 7.0 to 92.3 , with a mean MVD of $42.1 \pm 15.0$. No significant difference was observed between PROX1 expression and MVD $(P=0.188)$. The LVD of the 327 tumor samples ranged from 3.8 to 15.5 , with a mean LVD of $8.9 \pm 2.7$. The mean LVD value of PROX1-positive tumors was $9.5 \pm 2.5$, which was significantly higher than that of PROX1-negative tumors $(P=0.031$; Table 3$)$.

\section{Discussion}

PROX1 performs both oncogenic and tumor-suppressive functions depending on the tumor in which it is expressed, and its expression is altered in various human cancers [8]. and SNU638 cells than among SS-transfected AGS and SNU638 cells ( $P=0.037$ and $P=0.048$, respectively). c The cell morphologies of PS- and SS-transfected cells were observed via phase-contrast microscopy. PS-transfected AGS and SNU638 cells displayed cobblestone-like morphologic features and growth in clusters, unlike SS-transfected AGS and SNU638 cells $(200 \times)$. d Expression of MMP-2, MMP-9, ZO-1, and vimentin was lower in PS-transfected AGS and SNU638 cells than in SS-transfected AGS and SNU638 cells. However, expression of E-cadherin and snail were not altered after $P R O X 1$ knockdown in all tested cells. SS scrambled siRNA, PS $P R O X 1$ siRNA, FN fibronectin; Col I collagen I, Col IV collagen IV, $M M P$ matrix metalloproteinase; $Z O$ zonula occludens

However, the distinct function of PROX1 in human gastric cancer is largely unclear.

A balance between cell growth and apoptosis is important for the preservation of normal homeostasis and morphogenesis of human tissues. Disruption of this balance provides a survival advantage to cancer cells and promotes tumor development and progression [18, 19]. We first investigated whether inactivation of PROX1 affected the fate of human gastric cancer cells. We observed that PROX1 knockdown induced apoptosis by upregulating the activities of caspases and by decreasing the expression of anti-apoptotic proteins $\mathrm{Bcl}-2$ and $\mathrm{Bcl}-\mathrm{xL}$. In addition, PROXI knockdown inhibited the proliferation of human gastric cancer cells. These results suggested that PROX1 played a potential role in regulating cell fate by inhibiting 
Table 1 Associations between PROX1 expression and clinicopathological parameters of patients with gastric cancer

\begin{tabular}{|c|c|c|c|c|}
\hline \multirow[t]{2}{*}{ Parameters } & \multirow{2}{*}{$\begin{array}{l}\text { Total } \\
(n=327)\end{array}$} & \multicolumn{2}{|l|}{ PROX1 } & \multirow[t]{2}{*}{$P$ value } \\
\hline & & $\begin{array}{l}\text { Negative } \\
(n=134)\end{array}$ & $\begin{array}{l}\text { Positive } \\
(n=193)\end{array}$ & \\
\hline Age (years) & & & & $<0.001$ \\
\hline$<58.7$ & 118 & 64 & 54 & \\
\hline$\geq 58.7$ & 209 & 70 & 139 & \\
\hline Sex & & & & 0.055 \\
\hline Male & 215 & 80 & 135 & \\
\hline Female & 112 & 54 & 58 & \\
\hline Tumor size $(\mathrm{cm})$ & & & & 0.051 \\
\hline$<4.3$ & 199 & 90 & 109 & \\
\hline$\geq 4.3$ & 128 & 44 & 84 & \\
\hline Histologic type & & & & 0.001 \\
\hline Differentiated & 140 & 43 & 97 & \\
\hline Undifferentiated & 187 & 91 & 96 & \\
\hline Stage & & & & 0.012 \\
\hline $\mathrm{I} / \mathrm{II}$ & 198 & 92 & 106 & \\
\hline III/IV & 129 & 42 & 87 & \\
\hline Depth of invasion $(\mathrm{T})$ & & & & 0.884 \\
\hline $\mathrm{T} 1 / \mathrm{T} 2$ & 179 & 74 & 105 & \\
\hline $\mathrm{T} 3 / \mathrm{T} 4$ & 148 & 60 & 88 & \\
\hline $\begin{array}{l}\text { Lymph node } \\
\text { metastasis }(\mathrm{N})\end{array}$ & & & & 0.002 \\
\hline NO & 176 & 86 & 90 & \\
\hline N1-3 & 151 & 48 & 103 & \\
\hline Distant metastasis (M) & & & & N/A \\
\hline M0 & 327 & 134 & 193 & \\
\hline M1 & 0 & 0 & 0 & \\
\hline
\end{tabular}

PROX1 prospero homeobox 1, N/A not available apoptosis and by promoting the proliferation of human gastric cancer cells.

Tumor angiogenesis and lymphangiogenesis are the key features of tumor progression and metastasis in various human cancers, including gastric cancer [20-24]. We assessed the impact of PROX1 expression on angiogenesisand lymphangiogenesis-promoting phenotypes of human gastric cancer cells by performing in vitro assays. We observed that incubation of HLECs in CM from PROXI siRNA-transfected AGS and SNU638 cells significantly decreased their invasive and tube-formation abilities. PROXI knockdown decreased the expression of lymphangiogenic inducers VEGF-C, VEGF-D, and COX-2 in human gastric cancer cells. Previous studies have shown that VEGF-C and VEGF-D induce the proliferation and migration of LECs by activating VEGFR-3, and that overexpression of VEGF-C, VEGF-D, or VEGFR-3 is associated with lymphatic metastasis and poor clinical outcomes of various cancers [25-27]. Further, COX-2 promotes tumor lymphangiogenesis and lymph node metastasis [28]. These results indicate that PROX1 may play an important role in promoting tumor progression by stimulating tumor lymphangiogenesis along with VEGF-C, VEGF-D, and COX-2 in human gastric cancer. However, PROX1 knockdown did not inhibit the tube formation ability of HUVECs, indicating that it did not affect angiogenesis.

EMT causes cells to lose their epithelial polarity and to gain migratory mesenchymal properties. There is increasing evidence that EMT is a major contributor to tumor progression and metastasis and is associated with poor clinical outcomes of various cancers [29, 30]. A study
Fig. 4 Kaplan-Meier survival curves for the correlations of overall survival with positive (dotted line) and negative (solid line) PROX1 expression. The overall survival of patients with PROX1-positive tumors was significantly lower than that of patients with PROX1-negative tumors $(P<0.001)$

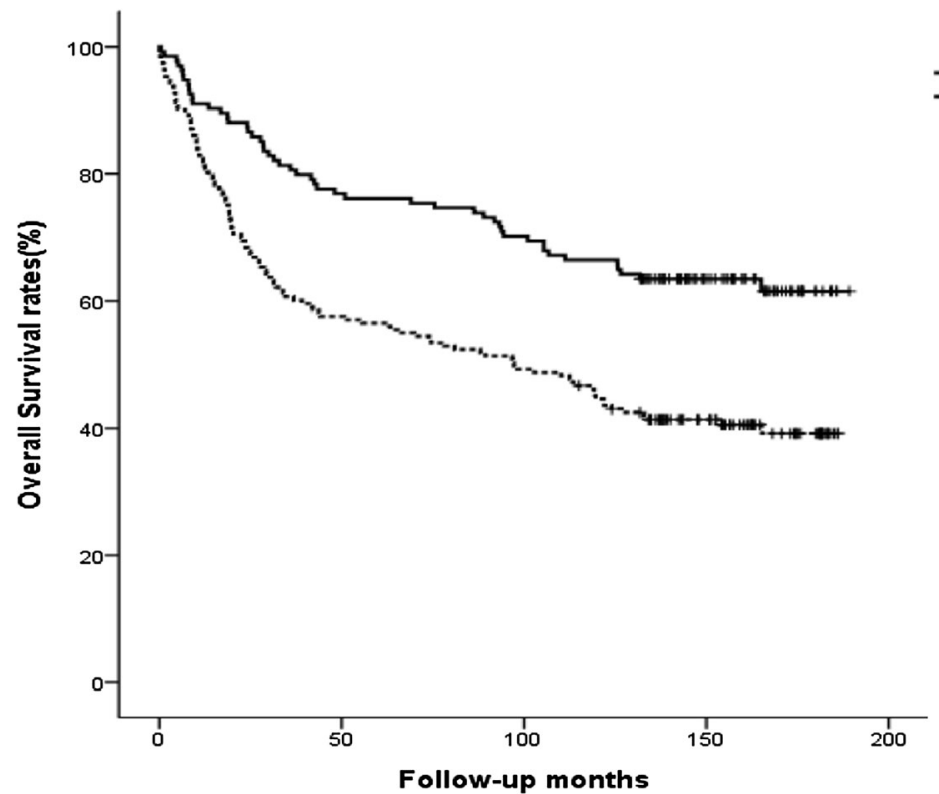

PROX1

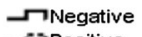
-.rpositive 
Table 2 Cox multivariate regression analysis of the association between PROX1 expression and survival of patients with gastric cancer

\begin{tabular}{|c|c|c|c|}
\hline Covariate & HR & $95 \%$ CI & $P$ value \\
\hline \multicolumn{4}{|l|}{ PROX1 expression } \\
\hline Low & 1.000 & Ref. & \\
\hline High & 1.662 & $1.180-2.339$ & 0.004 \\
\hline \multicolumn{4}{|l|}{ Age } \\
\hline$<58.7$ & 1.000 & Ref. & \\
\hline$\geq 58.7$ & 1.037 & $1.020-1.054$ & $<0.001$ \\
\hline \multicolumn{4}{|l|}{ Sex } \\
\hline Male & 1.000 & Ref. & \\
\hline Female & 1.419 & $1.012-1.989$ & 0.043 \\
\hline \multicolumn{4}{|l|}{ Tumor size } \\
\hline$<4.3$ & 1.000 & Ref. & \\
\hline$\geq 4.3$ & 1.356 & $0.969-1.896$ & 0.075 \\
\hline \multicolumn{4}{|l|}{ Histologic type } \\
\hline Differentiated & 1.000 & Ref. & \\
\hline Undifferentiated & 1.343 & $0.965-1.868$ & 0.080 \\
\hline \multicolumn{4}{|l|}{ Stage } \\
\hline I/II & 1.000 & Ref. & \\
\hline III/IV & 4.077 & $2.874-5.784$ & $<0.001$ \\
\hline
\end{tabular}

PROX1 prospero homeobox $1, H R$ hazard ratio, CI confidence interval, Ref. reference in Cox proportional hazards model

indicated that PROX1 promoted EMT and tumor progression by downregulating E-cadherin and upregulating MMPs in colorectal cancer cells [31]. Our study showed that PROXI knockdown increased the adhesion of gastric cancer cells to ECM proteins and decreased their migration. Although PROXI knockdown did not alter the expression of the epithelial marker E-cadherin, it decreased the expression of the migratory mesenchymal markers MMP-2, MMP-9, ZO-1, and vimentin. These results indicated that PROX1 induced phenotypic alterations similar to those observed during EMT, which are crucial for the progression and metastasis of human gastric cancer.

Next, we studied the effects of PROX1 on the stimulation of multiple intracellular signaling pathways involved in apoptosis, proliferation, angiogenesis, lymphangiogenesis, and EMT of gastric cancer cells to determine the mechanisms underlying the effects of PROX1. Wnt/ $\beta$-catenin/Tcell factor (TCF) and MAPK signaling pathways are involved in the migration, adhesion, proliferation, survival, EMT, angiogenesis, and lymphangiogenesis of various human cancer cells $[32,33]$. We observed that phosphorylation of $\beta$-catenin was increased and that of ERK1/2, p38, and JNK was decreased after PROX1 knockdown. A study showed that the $\mathrm{Wnt} / \beta$-catenin/TCF signaling pathway controls PROX1 expression in colorectal cancer cells that results in the neoplastic transformation of these cells, thus indicating an oncogenic role of PROX1 in colorectal cancer. However, this pathway does not control PROX1 expression in hepatocellular carcinoma cells, indicating a tumor-suppressive role of PROX1 in this cancer [34]. These contradictory results indicate tissue-specific differences in signaling pathways in different cancer types. However, the relationship between PROX1 and the Wnt/ $\beta$-catenin/TCF signaling pathway in gastric cancer remains unknown. These results suggest that PROX1 may activate the Wnt/ $\beta$-catenin/ TCF signaling pathway in gastric cancer, thus performing an oncogenic role.

Next, we evaluated PROX1 expression in a well-defined series of human gastric cancers, including long-term and complete follow-up, with special reference to patient prognosis. In our study, PROX1 protein was predominantly immunostained in the cytoplasm of gastric cancer cells. Previously, immunostaining of PROX1 protein showed both nuclear and cytoplasmic expression in gastric cancer, and cytoplasmic expression of PROX1 protein correlated with its mRNA amplification [35]. However, in colorectal cancer, expression of PROX1 protein was limited to tumor cell nuclei [13]. PROX1 is a transcription factor and its level and subcellular localization may be dynamically changed during carcinogenesis. Therefore, the subcellular localization of PROX1 is still contradictory and may be tissue dependent.

We observed that PROX1 expression was increased in human gastric cancer and metastatic lymph node tissues compared with that in normal gastric mucosa and nonmetastatic lymph node tissues. PROX1 expression was
Table 3 Associations of PROX1 expression with apoptosis, proliferation, angiogenesis, and lymphangiogenesis of human gastric cancer cells

\begin{tabular}{lcccc}
\hline Parameter (mean \pm SD) & Total $(n=327)$ & PROX1 expression & $P$ value \\
\cline { 3 - 4 } & & Negative $(n=134)$ & Positive $(n=193)$ & \\
\hline AI & $1.8 \pm 1.4$ & $1.6 \pm 1.5$ & $2.1 \pm 1.3$ & 0.381 \\
KI & $49.8 \pm 17.8$ & $45.3 \pm 18.5$ & $60.0 \pm 15.9$ & 0.002 \\
MVD & $42.1 \pm 15.0$ & $39.8 \pm 14.7$ & $44.6 \pm 15.2$ & 0.188 \\
LVD & $8.9 \pm 2.7$ & $8.4 \pm 2.8$ & $9.5 \pm 2.5$ & 0.031 \\
\hline
\end{tabular}

PROX1 prospero homeobox 1, $A I$ apoptotic index, $K I$ Ki-67 labeling index, $M V D$ microvessel density, $L V D$ lymphatic vessel density, $S D$ standard deviation 
significantly associated with age, cell differentiation, cancer stage, lymph node metastasis, and poor survival. In addition, patients with PROX1-positive tumors showed an elevated risk of death after adjustment for age, sex, and tumor size. A previous study showed that PROX1 expression was correlated with tumor grade and regional lymph node metastasis in human gastric cancer [35]. These results suggest that PROX1 plays an important role in tumor progression and may serve as a potential prognostic factor and target for treating gastric cancer.

Finally, we evaluated the correlation of PROX1 expression with apoptosis, proliferation, angiogenesis, and lymphangiogenesis in human gastric cancer tissues to confirm the results obtained from our in vitro experiments. We observed that the mean KI and LVD values of PROX1positive tumors were significantly higher than those of PROX1-negative tumors. However, no significant difference was observed between PROX1 expression and AI or MVD value. These results thus confirm the inhibition of the proliferation- and lymphangiogenesis-inducing potential of PROX1 knockdown in vivo.

Taken together, our results indicate that PROX1 promotes tumor progression by inducing the proliferation and lymphangiogenesis of gastric cancer cells.

\section{Compliance with ethical standards}

Conflict of interest All authors declare that they have no conflict of interest.

Ethical standards All procedures followed were in accordance with the ethical standards of the responsible committee on human experimentation (institutional and national) and with the Helsinki Declaration of 1964 and later versions. This study was approved by the Institutional Review Board of the Chonnam National University Hwasun Hospital. Also, ethical approval was obtained from the Institutional Review Board of the Chonnam National University Hwasun Hospital.

\section{References}

1. Guggenheim DE, Shah MA. Gastric cancer epidemiology and risk factors. J Surg Oncol. 2013;107:230-6.

2. Krejs GJ. Gastric cancer: epidemiology and risk factors. Dig Dis. 2010;28:600-3.

3. Gan L, Xu M, Zhang Y, Zhang X, Guo W. Focusing on long noncoding RNA dysregulation in gastric cancer. Tumour Biol. 2015;36:129-41.

4. Jin X, Zhu Z, Shi Y. Metastasis mechanism and gene/protein expression in gastric cancer with distant organs metastasis. Bull Cancer. 2014;101:E1-12.

5. Brábek J, Mierke CT, Rösel D, Veselý P, Fabry B. The role of the tissue microenvironment in the regulation of cancer cell motility and invasion. Cell Commun Signal. 2010;8:22.

6. Chaffer CL, Weinberg RA. A perspective on cancer cell metastasis. Science. 2011;331:1559-64.
7. Northcott JM, Northey JJ, Barnes JM, Weaver VM. Fighting the force: potential of homeobox genes for tumor microenvironment regulation. Biochim Biophys Acta. 2015;1855:248-53.

8. Elsir T, Smits A, Lindström MS, Nistér M. Transcription factor PROX1: its role in development and cancer. Cancer Metastasis Rev. 2012;31:793-805.

9. Schneider M, Büchler P, Giese N, Giese T, Wilting J, Büchler MW, et al. Role of lymphangiogenesis and lymphangiogenic factors during pancreatic cancer progression and lymphatic spread. Int J Oncol. 2006;28:883-90.

10. Shimoda M, Takahashi M, Yoshimoto T, Kono T, Ikai I, Kubo H. A homeobox protein, prox1, is involved in the differentiation, proliferation, and prognosis in hepatocellular carcinoma. Clin Cancer Res. 2006;12:6005-11.

11. Versmold B, Felsberg J, Mikeska T, Ehrentraut D, Köhler J, Hampl JA, et al. Epigenetic silencing of the candidate tumor suppressor gene PROX1 in sporadic breast cancer. Int J Cancer. 2007;121:547-54.

12. Laerm A, Helmbold P, Goldberg M, Dammann R, Holzhausen HJ, Ballhausen WG. Prospero-related homeobox 1 (PROX1) is frequently inactivated by genomic deletions and epigenetic silencing in carcinomas of the biliary system. J Hepatol. 2007;46:89-97.

13. Skog M, Bono P, Lundin M, Lundin J, Louhimo J, Linder N, et al. Expression and prognostic value of transcription factor PROX1 in colorectal cancer. Br J Cancer. 2011;105:1346-51.

14. Miettinen M, Wang ZF. Prox 1 transcription factor as a marker for vascular tumors-evaluation of 314 vascular endothelial and 1086 nonvascular tumors. Am J Surg Pathol. 2012;36:351-9.

15. Elsir T, Qu M, Berntsson SG, Orrego A, Olofsson T, Lindström MS, et al. PROX1 is a predictor of survival for gliomas WHO grade II. Br J Cancer. 2011;104:1747-54.

16. Elsir T, Eriksson A, Orrego A, Lindström MS, Nistér $M$. Expression of PROX1 is a common feature of high-grade malignant astrocytic gliomas. J Neuropathol Exp Neurol. 2010;69:129-38.

17. Greene FL, American Joint Committee on Cancer, American Cancer Society. AJCC cancer staging manual. New York: Springer; 2002. p. xiv

18. Kiechle FL, Zhang X. Apoptosis: biochemical aspects and clinical implications. Clin Chim Acta. 2002;326:27-45.

19. Schultz DR, Harrington WJ Jr. Apoptosis: programmed cell death at a molecular level. Semin Arthritis Rheum. 2003;32:345-69.

20. Mittal K, Ebos J, Rini B. Angiogenesis and the tumor microenvironment: vascular endothelial growth factor and beyond. Semin Oncol. 2014;41:235-51.

21. Gomes FG, Nedel F, Alves AM, Nör JE, Tarquinio SB. Tumor angiogenesis and lymphangiogenesis: tumor/endothelial crosstalk and cellular/microenvironmental signaling mechanisms. Life Sci. 2013;92:101-7.

22. Stacker SA, Williams SP, Karnezis T, Shayan R, Fox SB, Achen MG. Lymphangiogenesis and lymphatic vessel remodelling in cancer. Nat Rev Cancer. 2014;14:159-72.

23. Kitadai Y. Angiogenesis and lymphangiogenesis of gastric cancer. J Oncol. 2010;468725. doi:10.1155/2010/468725.

24. Sarvaiya PJ, Guo D, Ulasov I, Gabikian P, Lesniak MS. Chemokines in tumor progression and metastasis. Oncotarget. 2013;4:2171-85.

25. Duong T, Koopman P, Francois M. Tumor lymphangiogenesis as a potential therapeutic target. J Oncol. 2012;204946. doi:10.1155/ 2012/204946.

26. Albrecht I, Christofori G. Molecular mechanisms of lymphangiogenesis in development and cancer. Int $\mathrm{J}$ Dev Biol. 2011:55:483-94.

27. Langheinrich MC, Schellerer V, Perrakis A, Lohmüller C, Schildberg C, Naschberger E, et al. Molecular mechanisms of 
lymphatic metastasis in solid tumors of the gastrointestinal tract. Int J Clin Exp Pathol. 2012;5:614-23.

28. Karnezis T, Shayan R, Fox S, Achen MG, Stacker SA. The connection between lymphangiogenic signalling and prostaglandin biology: a missing link in the metastatic pathway. Oncotarget. 2012;3:893-906.

29. Steinestel K, Eder S, Schrader AJ, Steinestel J. Clinical significance of epithelial-mesenchymal transition. Clin Transl Med. 2014;3:17. doi:10.1186/2001-1326-3-17.

30. Guarino M, Rubino B, Ballabio G. The role of epithelial-mesenchymal transition in cancer pathology. Pathology. 2007;39:305-18.

31. Lu MH, Huang CC, Pan MR, Chen HH, Hung WC. Prospero homeobox 1 promotes epithelial-mesenchymal transition in colon cancer cells by inhibiting E-cadherin via miR-9. Clin Cancer Res. 2012;18:6416-25.

32. Ring A, Kim YM, Kahn M. Wnt/catenin signaling in adult stem cell physiology and disease. Stem Cell Rev. 2014;10:512-25.

33. Haagenson $\mathrm{KK}, \mathrm{Wu}$ GS. Mitogen activated protein kinase phosphatases and cancer. Cancer Biol Ther. 2010;9:337-40.

34. Petrova TV, Nykänen A, Norrmén C, Ivanov KI, Andersson LC, Haglund C, et al. Transcription factor PROX1 induces colon cancer progression by promoting the transition from benign to highly dysplastic phenotype. Cancer Cell. 2008;13:407-19.

35. Taban O, Cimpean AM, Raica M, Olariu S. PROX1 expression in gastric cancer: from hypothesis to evidence. Anticancer Res. 2014;34:3439-46. 